\title{
Recomendaciones para la atención de pacientes con insuficiencia cardiaca y COVID-19
}

\author{
Recommendations for the care of patients with heart failure and COVID-19
}

José A. Cigarroa-López ${ }^{1,2,3 *}$, José A. Magaña-Serrano ${ }^{1,2,3}$, Amada Álvarez-Sangabriel2,4, Vicente Ruíz-Ruízz ${ }^{2}$, Adolfo Chávez-Mendozaa ${ }^{1,2,3}$, Arturo Méndez-Ortíz ${ }^{2,4}$, Salvador León-González ${ }^{2,5}$, Carlos Guízar-Sánchez ${ }^{2,4}$, Pedro Gutiérrez-Fajardo y Marco A. Alcocer-Gamba ${ }^{2,5}$

${ }^{1}$ Asociación Nacional de Cardiólogos de México; ${ }^{2}$ Sociedad Mexicana de Cardiología; ${ }^{3}$ División de Insuficiencia y Trasplante de la UMAE H. Cardiología del CMN Siglo XXI. IMSS; ${ }^{4}$ Insuficiencia Cardiaca y Trasplante Cardiaco, Instituto Nacional de Cardiología "Ignacio Chávez"; ${ }^{5}$ Instituto de Corazón de Querétaro

\begin{abstract}
Resumen
La pandemia por COVID-19 decretada por la Organización Mundial de la Salud (OMS) desde el 12 de marzo de 2020 está produciendo estragos a nivel mundial y es un verdadero reto económico, social y sanitario. Aunque las manifestaciones clínicas del COVID-19 son síntomas respiratorios, algunos pacientes también tienen síntomas cardiológicos. Dentro de los pacientes con afecciones cardiológicas2 suponen un grupo de mayor riesgo y que de hecho son un grupo especialmente vulnerable, por su mayor riesgo de contagio y mayor gravedad en caso de adquirir la enfermedad1 aquellos con insuficiencia cardiaca (IC), incluyendo al trasplante cardiaco (TC) y las asistencias ventriculares, así como los pacientes con hipertensión arterial pulmonar (HAP). La IC es la principal patología cardiovascular crónica y los pacientes en este grupo son los más vulnerables para el desarrollo de cuadros clínicos más graves tras sufrir la infección, y en mayor medida los casos con IC avanzada3. De hecho, la IC es unas de las complicaciones más frecuentes en los pacientes con COVID-194. De igual forma, los pacientes trasplantados que requieren de los inmunosupresores para evitar el rechazo del injerto, constituyen una población especialmente susceptible a la infección y a desarrollar procesos más graves. Esta situación ha hecho que la Asociación Nacional de Cardiólogos de México (ANCAM) y la Sociedad Mexicana de Cardiología (SMC) junto con sus respectivos capítulos, hayan elaborado las siguientes recomendaciones para el personal médico, que participa en la atención de este grupo especial de pacientes en los diferentes escenarios clínicos, que padezcan o no, COVID-19.
\end{abstract}

Palabras clave: Insuficiencia cardiaca. COVID-19. Pandemia. Cardiología. Hipertensión arterial pulmonar. Sociedad Mexicana de Cardiología. Asociación Nacional de Cardiólogos de México.

\begin{abstract}
The COVID-19 pandemic decreed by the World Health Organization (WHO) since March 12, 2020 is wreaking havoc globally and it is a true economic, social and health challenge. Although the clinical manifestations of COVID-19 are
\end{abstract}

\section{Correspondencia:}

*José A. Cigarroa-López

E-mail: drangelcigarroa@gmail.com
Disponible en internet: $26-05-2020$ Arch Cardiol Mex. 2020;90(Supl):26-32 www.archivoscardiologia.com 1405-9940/@ 2020 Instituto Nacional de Cardiología Ignacio Chávez. Publicado por Permanyer. Este es un artículo open access bajo la licencia CC BY-NC-ND (http://creativecommons.org/licenses/by-nc-nd/4.0/). 
respiratory symptoms, some patients also have cardiological symptoms. Among patients with cardiological conditions2 they represent a group of higher risk and, in fact, they are a particularly vulnerable group, due to their higher risk of contagion and greater severity in case of acquiring the disease 1 those with heart failure (HF), including heart transplant (CT) and ventricular assists, as well as patients with pulmonary arterial hypertension (PAH). HF is the main chronic cardiovascular disease and patients in this group are the most vulnerable for the development of more serious clinical symptoms after suffering the infection, and to a greater extent cases with advanced HF3. In fact, HF is one of the most frequent complications in patients with COVID-194. Likewise, transplant patients who require immunosuppressants to avoid graft rejection, constitute a population especially susceptible to infection and to develop more serious processes. This situation has made the National Association of Cardiologists of Mexico (ANCAM) and the Mexican Society of Cardiology (SMC) together with their respective chapters, have prepared the following recommendations for medical personnel, who participate in the care of this special group of patients in the different clinical settings, who suffer or not, of COVID-19.

Key words: Heart failure. COVID-19. Pandemic. Cardiology. Pulmonary arterial hypertension. Mexican Society of Cardiology. National Association of Cardiologists of Mexico.

La pandemia por COVID-19 decretada por la Organización Mundial de la Salud (OMS) desde el 12 de marzo de 2020, está produciendo estragos a nivel mundial y es un verdadero reto económico, social y sanitario. Aunque las manifestaciones clínicas del COVID-19, son síntomas respiratorios, algunos pacientes también tienen síntomas cardiológicos. Dentro de los pacientes con afecciones cardiológicas ${ }^{2}$, suponen un grupo de mayor riesgo y que de hecho son un grupo especialmente vulnerable, por su mayor riesgo de contagio y mayor gravedad en caso de adquirir la enfermedad ${ }^{1}$ aquellos con insuficiencia cardiaca (IC), incluyendo al trasplante cardiaco (TC) y las asistencias ventriculares, así como los pacientes con hipertensión arterial pulmonar (HAP). La IC es la principal patología cardiovascular crónica y los pacientes en este grupo son los más vulnerables para el desarrollo de cuadros clínicos más graves tras sufrir la infección y en mayor medida los casos con IC avanzada ${ }^{3}$. De hecho, la IC es unas de las complicaciones más frecuentes en los pacientes con COVID-194. De igual forma, los pacientes trasplantados, que requieren de los inmunosupresores para evitar el rechazo del injerto, constituyen una población especialmente susceptible a la infección y a desarrollar procesos más graves. Esta situación ha hecho que la Asociación Nacional de Cardiólogos de México (ANCAM) y la Sociedad Mexicana de Cardiología (SMC) junto con sus respectivos capítulos, hayan elaborado las siguientes recomendaciones para el personal médico, que participa en la atención de este grupo especial de pacientes en los diferentes escenarios clínicos, que padezcan o no, COVID-19.

\section{Pacientes ambulatorios con insuficiencia cardiaca en el contexto de la pandemia por COVID-19}

Dado que la población con IC tiene un mayor riesgo de infección y peor pronóstico si cursan con COVID-19, sugerimos las siguientes medidas ${ }^{5}$ :

1. En todo paciente con IC, es necesario el aislamiento social y trabajar desde casa, durante todo el tiempo de la emergencia sanitaria.

2. Procurar establecer consulta a distancia y limitar al máximo las visitas presenciales en el consultorio $u$ hospital durante la duración de la contingencia sanitaria. El apoyo de enfermería en el seguimiento es fundamental, así como complementar la visita con la obtención de los datos que el paciente pueda realizar en su domicilio (disnea, edema, presión arterial, peso, frecuencia cardiaca, saturación de oxígeno $\left(\mathrm{SaO}_{2}\right)$, temperatura, etc.)

3. No cambiar o suspender el tratamiento de base en estos pacientes, especialmente cuando la indicación del mismo es la mejoría pronóstica, como en el caso de la IC con fracción de expulsión reducida. Importante enfatizar en la continuidad del tratamiento con IECA/ARA2/Sacubitril-Valsartán y en el recomendado de acuerdo con guías.

4. En caso de que el paciente, cuente con un dispositivo implantado (DAl, marcapasos, o resincronizador), procurar revisarlos por telemetría. Este tipo de monitoreo remoto permite comprobar el adecuado funcionamiento de los dispositivos, así como revisar eventos arrítmicos y eventuales descargas. Cuando no se pueda y sea absolutamente necesaria la revisión 
Arch Cardiol Mex. 2020;90(Supl)

Tabla 1. Diferencias entre insuficiencia cardiaca descompensada e infección por COVID-19

\begin{tabular}{|c|c|c|}
\hline & COVID-19 & Insuficiencia cardiaca \\
\hline \multicolumn{3}{|l|}{ Analítica } \\
\hline Linfopenia & +++ & - \\
\hline ProBNP & $-1+$ & +++ \\
\hline PCR & +++ & + \\
\hline D-Dímero & +++ & $-1+$ \\
\hline \multicolumn{3}{|l|}{ Pruebas de imagen } \\
\hline \multicolumn{3}{|l|}{ Radiografia Tórax } \\
\hline Infiltrados & Periféricos & Centrales, en alas de mariposa \\
\hline Hilios aumentados & $/^{*}$ & +++ \\
\hline Cardiomegalia & $\cdots$ & + \\
\hline Derrame pleural & $\stackrel{*}{*}$ & + \\
\hline$T A C$ & $\begin{array}{l}\text { Imágenes en vidrio deslustrado de } \\
\text { predominio periférico bilaterales (al inicio } \\
\text { pueden ser unilaterales) } \\
\text { +/- Condensaciones segmentarias } \\
\text { +/- engrosamiento pleural }\end{array}$ & $\begin{array}{l}\text { Imágenes en vidrio deslustrado de } \\
\text { predominio central } \\
\text { Condensaciones de predominio central } \\
\text { Dilatación de venas pulmonares } \\
\text { Derrame pleural }\end{array}$ \\
\hline
\end{tabular}

* Puede estar presente en IC previa

Tomado de Bugadá y Cols. ${ }^{5}$

presencial, se deberá acudir al consultorio u hospital y evitar la estancia en el servicio de urgencias.

5. Si el paciente presenta datos de descompensación, procurar la atención en domicilio o en su defecto, utilizar la corta estancia u "hospital de día" para evitar la hospitalización. En caso de requerirse hospitalización, De acuerdo con los criterios establecidos, deberá procurarse que sea en un ambiente libre de infección por Covid-19.

\section{Paciente hospitalizado por descompensación de insuficiencia cardiaca}

Si el paciente con IC tiene una descompensación y requiere hospitalización, después de haber agotado los recursos ambulatorios, se deben buscar dos objetivos: a) evitar el contagio del virus, y b) mejorar la IC lo más pronto posible. Para ello se requiere:

1. Planificar desde antes de su ingreso al hospital, la terapia a proporcionar.

2. Gestionar que su ingreso sea directo a hospitalización, preferentemente sin pasar por el servicio de urgencias

3. Hospitalizarlo en zona libre de COVID-19 (establecido por cada unidad médica).

4. Permanecer en la habitación con un máximo de un acompañante y sin visitas.

5. Únicamente se realizarán exámenes de laboratorio o gabinete que se consideren imprescindibles (péptidos natriuréticos, biometría hemática $(\mathrm{BH})$, electrolitos séricos, gasometría y azoados, entre otros), para la estratificación y manejo del paciente. Deben evitarse exámenes y procedimientos que no determinen el tratamiento inmediato, que puedan prolongar la estancia hospitalaria y que se pueden realizar en forma externa.

6. En cuanto sea factible, se deben plantear estrategias de alta temprana con soporte de terapias intermitentes mediante hospitalización a domicilio, visitas en domicilio 0 atención telefónica por parte del personal médico de insuficiencia cardiaca para disminuir el tiempo de estancia en zonas con alto riesgo de contagio y liberar camas de hospitalización.

7. Establecer el diagnóstico de sospecha de infección por COVID-19 ante condiciones clínicas o de laboratorio (NT proBNP, Dímero D, PCR de alta sensibilidad, Troponinas, entre otros).

\section{Paciente ingresado con insuficiencia cardiaca y sospecha/infección por COVID-19}

Es importante considerar que el paciente con IC y sospecha o confirmación de COVID-19 tiene un pronóstico adverso, debido a que la infección por este virus agrava la enfermedad de base e incluso se puede agregar daño miocárdico directo que podría evolucionar a falla multiorgánica o miocarditis fulminante. El daño miocárdico y la IC con o sin insuficiencia 
respiratoria representa hasta el $40 \%$ de mortalidad de estos pacientes.

En caso de síntomas de evolución rápida y progresivos de descompensación de IC, más la presencia de fiebre y tos, deberá realizarse la prueba para determinar COVID-19. Una vez confirmado, iniciar protocolo de aislamiento y exámenes de laboratorio pertinentes, además de determinación de Troponinas (Tn), NT proBNP, Dímero D y LDH, Proteina $C$ reactiva (PCR) y Pro calcitonina; en el caso de que la prueba para SARS-CoV-2 fuera negativa (lo cual puede ser en los primeros 3 días de iniciados los síntomas), repetirla a las 72 horas y tratar al paciente como positivo hasta su confirmación. En relación a los estudios de imagen, es importante realizar una radiografía de tórax e incluso una tomografía de tórax para valorar la severidad de la neumonía por COVID-19.

En tanto se tenga la confirmación de la prueba para SARS-CoV-2, valorar los síntomas o signos del paciente con IC para poder encaminar el diagnóstico y en ese sentido poder guiar el tratamiento de la descompensación de la enfermedad de base; si el paciente presenta síntomas virales y/o fiebre es muy probable que tenga COVID-19, si el paciente tiene tos, fatiga y disnea puede tratarse de COVID-19 o descompensación de la IC. Si por el contrario al paciente le aumenta el edema, la ascitis o la ganancia de peso, orienta más a la probabilidad de agudización de la IC.

En el manejo del paciente con IC y COVID-19, debe tenerse una conducta conservadora en relación a la administración de soluciones parenterales. En caso de datos de hipoperfusión, se recomienda la monitorización invasiva de parámetros hemodinámicos. Si existe insuficiencia respiratoria refractaria a la administración de oxígeno suplementario, considerar un modo de ventilación no invasiva que no genere dispersión del aire exhalado. En los casos donde el paciente presente edema agudo de pulmón (EAP) se prefiere no retrasar la intubación endotraqueal, favoreciendo protocolos de ventilación para distrés respiratorio. En aquellos casos donde no se logra mejorar la oxigenación con ventilación mecánica podrá considerarse la utilización de ECMO.

Algunos medicamentos utilizados para el tratamiento de COVID-19, pueden generar arritmias ventriculares, por lo que es importante hacer las consideraciones necesarias, particularmente medición del QT y QTc, así como niveles de electrólitos séricos para evitar efectos adversos potencialmente fatales.
Tabla 2. Ajustes al tratamiento inmunosupresor del paciente trasplantado

\begin{tabular}{|l|l|}
\hline Infección leve & $\begin{array}{c}\text { - Mantener tratamiento habitual de } \\
\text { inmunosupresión o reducir a un nivel } \\
\text { sérico inferior terapéutico. } \\
\text { - Suspender micofenolato o azatioprina } \\
\text { por } 48 \text { h y revalorar el inicio. }\end{array}$ \\
\hline $\begin{array}{l}\text { Infección } \\
\text { moderada a severa }\end{array}$ & $\begin{array}{l}\text { - Considerar suspender micofenolato/ } \\
\text { azatioprina y reducir niveles de } \\
\text { anticalcineurínico. }\end{array}$ \\
\hline $\begin{array}{l}\text { Se puede aumentar la terapia con } \\
\text { corticoesteroides o incluso administrar } \\
\text { inmunoglobulinas. }\end{array}$ \\
\hline $\begin{array}{l}\text { Tratamiento } \\
\text { farmacológico }\end{array}$ \\
$\begin{array}{l}\text { No existe evidencia específica de } \\
\text { ningún tratamiento hasta este } \\
\text { momento y se deben establecer } \\
\text { protocolos en cada centro. }\end{array}$ \\
\hline
\end{tabular}

\section{Paciente trasplantado de corazón}

Estas recomendaciones se emiten tomando en cuenta la evidencia actual y de acuerdo con los lineamientos de la International Society for Heart and Lung Transplantation (ISHLT), la Sociedad Europea de Cardiología, la Asociación Española de Insuficiencia Cardiaca, la Sociedad Española de Cardiología, así como por la Secretaría de Salud a través del Centro Nacional de Trasplantes (CENATRA) ${ }^{6}$.

1. Se recomienda en todo paciente trasplantado, aplicar medidas estrictas de aislamiento, y siempre que sea posible, fomentar el trabajo en casa y en caso de requerirlo, realizar la nota médica para el trabajo, a todos los pacientes que no puedan evitar el contacto con otras personas en su actividad laboral.

2. Se aconseja que todas las consultas posibles, se hagan vía telefónica o por video llamada.

3. En la medida de lo posible, se deberán posponer las biopsias endomiocárdicas, ecocardiogramas de seguimiento o estudios electivos, para evitar acudir al hospital.

4. Se aconseja mantener las biopsias endomiocárdicas o controles clínicos y analíticos solo dentro de los 3 primeros meses posteriores al trasplante 0 tras un episodio de rechazo. En caso contrario, se deben diferir.

5. Para los pacientes que tengan que acudir al hospital, se recomienda contacto telefónico previo para descartar síntomas sugestivos de infección y tomar las medidas necesarias si hay sospecha de infección por COVID-19.

6. Se debe buscar atender a los pacientes trasplantados en zonas libres de COVID-19 y tanto el paciente 
como el personal que les atienda, deberán llevar mascarilla de triple capa, como prevención.

7. Se debe evitar al máximo que el paciente salga de su casa y acuda al hospital.

\section{Paciente trasplantado con sospecha/ confirmación de infección por COVID-19}

Se deben realizar los estudios de imagen torácica, dándole preferencia a laomografía. Debe realizarse la prueba para determinar COVID-19, en cuanto se tenga la mínima sospecha de infección.

Con relación al tratamiento inmunosupresor, se sugieren los ajustes definidos en la tabla 2.

\section{Pacientes con insuficiencia cardiaca avanzada y necesidad de trasplante cardiaco}

Se recomienda evaluar cuidadosamente el riesgo-beneficio de realizar un trasplante cardiaco durante la emergencia sanitaria por COVID-19. Realizarlo, solo en casos de urgencia y maximizar el estudio del donante y del receptor con negatividad de frotis para SARS-CoV-2.

Para pacientes que han tenido infección por COVID-19, antes de proceder al trasplante, se recomienda esperar 14 días para el trasplante tras el diagnóstico inicial y tener dos frotis negativos, separados una semana. Debe confirmarse que no hay afección pulmonar por COVID-19 (mediante TAC) y confirmar con nuevos frotis seriados negativos. En todo caso, por el riesgo de fibrosis, se debe actuar con gran prudencia en este contexto.

\section{Pacientes con hipertensión arterial pulmonar y COVID-19}

Debido al riesgo incrementado, todo paciente con HAP durante la emergencia sanitaria debe mantener el aislamiento social y trabajar desde casa, así como seguir las medidas higiénicas recomendadas.

En la actualidad el aspecto más desafiante de la atención continua de los pacientes con HAP es considerar el "riesgo de exposición" de aquellos que acuden a la clínica u hospital para seguimiento y nuevas citas, incluidas las pruebas de laboratorio de rutina. Encontrar medios para agilizar la atención ambulatoria durante este tiempo y descargar el trabajo a los médicos mediante la utilización de médicos y enfermeras especializados, video llamadas, así como servicios de farmacia especializados, puede mejorar en gran medida los puntos de contacto con los pacientes para optimizar la detección de los signos de empeoramiento del estado clínico y de riesgo de estos pacientes. La atención se centra en los siguientes puntos:

1. Recibir nuevos pacientes para el diagnóstico y tratamiento de HAP, continúa requiriendo de una evaluación exhaustiva para excluir cuidadosamente los grupos 2 y 3 de hipertensión pulmonar (HP) antes de comenzar las terapias específicas para HAP9 .

2. Se tendrán que ponderar los riesgos de exposición a COVID-19 durante los procedimientos electivos, como el cateterismo cardíaco derecho (CCD) y los beneficios de esta evaluación para facilitar las terapias especificas para HAP.

3. Para pacientes estables con alta probabilidad de tener HP del Grupo 2 y 3, la evaluación electiva debe diferirse hasta una fecha ya que el abordaje diagnóstico y terapéutico deberá enfocarse al grupo de riesgo.

4. Iniciar y monitorizar las terapias de HAP puede ser manejado por enfermeras especializadas en HAP y llamadas/visitas telefónicas o video llamadas, en centros seleccionados.

5. El tratamiento de la HPTEC probablemente será similar al de la HAP durante la pandemia de COVID-19, considerando que la intervención quirúrgica y la angioplastia pulmonar con balón estarán menos disponibles de inmediato.

6. Los pacientes con HAP y HPTEC se atenderán solo si presentan IC derecha descompensada refractaria.

7. En pacientes que reciben anticoagulación para HPTE y fibrilación auricular, entre otros, se sugiere cambiar a anticoagulantes orales directos o heparina de bajo peso molecular para evitar la visita al laboratorio para obtener y controlar un INR terapéutico con uso de warfarina o acenocumarina.

8. En pacientes inestables en la UCI, con COVID-19, utilizar oxigenoterapia de alto flujo, ventilación mecánica (BiPAP/CPAP), intubación y, en algunos casos, ECMO. En este contexto, el manejo de la ventilación y el soporte circulatorio presentan desafíos únicos y muy complejos, que limitan la supervivencia del paciente, por lo que se debe considerar la utilización de una herramienta de evaluación de riesgos específica de HAP establecida, para ayudar a identificar a los pacientes que tienen más probabilidades de sobrevivir a estas intervenciones durante el brote de COVID-19.10-13.

9. En pacientes con HAP y empeoramiento de la función cardiaca derecha, el diagnóstico diferencial incluye sepsis, isquemia, progresión de la 
enfermedad, tromboembolia pulmonar o infección por COVID-19 (o una combinación de estos factores).

10. Durante la pandemia actual, se debe suponer que la fiebre en el hogar de un paciente con HAP, representa una infección por COVID-19. Si los síntomas respiratorios de un paciente están empeorando y requiere de hospitalización, debe evaluarse y analizarse para detectar COVID-19.

11. Hay datos sobre el riesgo de síndrome de distrés respiratorio agudo (SDRA) en la enfermedad vascular pulmonar que sugieren que la neumonía por COVID-19 en el contexto de la HAP resultará más común en SDR ${ }^{14,15}$

12. En general, las terapias específicas de HAP deben continuarse durante la hospitalización y los pacientes que no pueden tolerar los medicamentos orales o inhalados pueden necesitar pasar de medicamentos orales a intravenosos para superar la gravedad de una infección por COVID-196.

\section{Conclusiones}

El tratamiento farmacológico especifico de COVID-19, no se ha definido con certeza y se están realizando múltiples ensayos clínicos para determinar eventualmente un tratamiento estandarizado. Es por ello, que por el momento cada centro hospitalario, debe definir su esquema. Es importante enfatizar que debemos extremar las precauciones de protección para disminuir al máximo el número de contagios, en especial al grupo mas vulnerable dentro de las cardiopatías que son los pacientes con IC, trasplantados y con HAP. Debemos evitar en lo posible las visitas hospitalarias y tratar de resolverlas mediante comunicación telefónica o video llamada. Cuando un paciente con IC se infecta por COVID-19, la IC se puede agravar tanto por la misma condición cardiológica, como por la propia infección. Es probable que la infección por COVID-19 y la falla cardiaca derecha concomitante conduzca a una mayor mortalidad en el paciente con HAP. El manejo de la ventilación y el soporte circulatorio presentan desafios únicos y muy complejos, que limitan la supervivencia del paciente, por lo que se debe considerar la utilización de una herramienta de evaluación de riesgos específica de HAP establecida para ayudar a identificar a los pacientes que tienen más probabilidades de sobrevivir a estas intervenciones durante el brote de COVID-19. Por lo anterior, debemos extremar las medidas de prevención y control de los pacientes durante la emergencia sanitaria y mantener optimizado el tratamiento farmacológico de nuestros pacientes. Esperemos que médicos, farmacéuticas, sociedades médicas, gobierno y sociedad, en conjunto, podamos combatir de manera eficiente esta pandemia y ofrecer las mejores conductas de prevención y terapéuticas para el bienestar de los pacientes en este grupo especial de muy alto riesgo.

\section{Responsabilidades éticas}

Protección de personas y animales. Los autores declaran que para esta investigación no se han realizado experimentos en seres humanos ni en animales.

Confidencialidad de los datos. Los autores declaran que han seguido los protocolos de su centro de trabajo sobre la publicación de datos de pacientes.

Derecho a la privacidad y consentimiento informado. Los autores declaran que en este artículo no aparecen datos de pacientes.

\section{Bibliografía}

1. Wang D, Hu B, Hu C, et al. Clinical characteristics of 138 hospitalized patients with 2019 novel coronavirus-infected pneumonia in Wuhan, China. JAMA. 2020. https://doi.org/10.1001/jama.2020.1585.

2. Chen N, Zhou M, Dong X, et al. Epidemiological and clinical characteristics of 99 cases of 2019 novel coronavirus pneumonia in Wuhan, China: a descriptive study. Lancet. 2020; 395:507-13.

3. Zheng YY, Ma YT, Zhang JY, Xie X. COVID-19 and the cardiovascular system. Nat Rev Cardiol. 2020; https://doi.org/10.1038/s41569-020-0360-5.

4. Zhou F, Yu T, Du R, et al. Clinical course and risk factors for mortality of adult inpatients with COVID- 19 in Wuhan, China: a retrospective cohort study. Lancet. 2020. https://doi.org/10.1016/S0140- 6736(20)30566-3. Erratum in: Lancet. 2020.https://doi.org/10.1016/S0140-6736(20)30606-1.

5. Bagudá JJ, Farrero TM, Recio MA, García-Cosío MD, et al. Implicaciones de la pandemia por COVID-19 para el paciente con insuficiencia cardiaca, trasplante cardiaco y asistencia ventricular. Recomendaciones de la Asociación de Insuficiencia Cardiaca de la Sociedad Española de Cardiología 2020.https://secardiologia.es/images/secciones/insuficiencia/ Implicaciones_de_la_pandemia_por_COVID-19_para_el_paciente_con insuficiencia cardiaca trasplante cardiaco y asistencia ventricular.pdf

6. Recomendaciones al Subsistema Nacional de Donación y Trasplantes sobre la infección asociada al SARS-CoV2 (Covid-19), publicada el 1 de abril de 2020. www.gob.mx/cenatra.

7. Torres A, Rivera A, García A, et al. Evaluación y tratamiento de la insuficiencia cardiaca durante la pandemia de COVID-19: resumen ejecutivo Recomendaciones del capítulo de falla cardiaca, trasplante e hipertensión pulmonar de la Asociación Colombiana de Cardiología y Cirugía Cardiovascular. Marzo 2020.

8. Krahn A, Bewick SJ, Chow Ch, et al. Guidance from the CCS COVID-19 rapid response team. Canadian Cardiovascular Society. Marzo 2020

9. Galie N, Humbert M, Vachiery JL, Gibbs S, Lang I, Torbicki A, et al. 2015 ESC/ERS Guidelines for the diagnosis and treatment of pulmonary hypertension: The Joint Task Force for the Diagnosis and Treatment of Pulmonary Hypertension of the European Society of Cardiology (ESC) and the European Respiratory Society (ERS): Endorsed by: Association for European Paediatric and Congenital Cardiology (AEPC), International Society for Heart and Lung Transplantation (ISHLT). Eur Heart J. 2016;37(1):67-119.

10. Benza RL, Gomberg-Maitland M, Elliott CG, Farber HW, Foreman AJ, Frost $A E$, et al. Predicting Survival in Patients With Pulmonary Arterial Hypertension: The REVEAL Risk Score Calculator 2.0 and Comparison With ESC/ERS-Based Risk Assessment Strategies. Chest. 2019;156(2):323-37

11. Humbert M, Sitbon O, Yaici A, Montani D, O'Callaghan DS, Jais X, et al. Survival in incident and prevalent cohorts of patients with pulmonary arterial hypertension. Eur Respir J. 2010;36(3):549-55.

12. Huang $C$, Wang $Y$, Li $X$, Ren $L$, Zhao J, Hu Y, et al. Clinical features of patients infected with 2019 novel coronavirus in Wuhan, China. Lancet. 2020;395(10223):497-506.

13. Guan WJ, Ni ZY, Hu Y, Liang WH, Ou CQ, He JX, et al. Clinical characteristics of coronavirus disease 2019 in China. N Engl J Med. 2020. 19. 
Arch Cardiol Mex. 2020;90(Supl)

14. Price LC, Wort SJ. Pulmonary hypertension in ARDS: inflammation matters! Thorax. 2017;72(5):396-7.

15. Pandolfi R, Barreira B, Moreno E, Lara-Acedo V, Morales-Cano D, Martinez-Ramas A, et al. Role of acid sphingomyelinase and IL-6 as mediators of endotoxin-induced pulmonary vascular dysfunction. Thorax 2017;72(5):460-71.

16. Pan IZ Carey JR, Jacobs JA, Dechand J Sessions JJ, Sorensen T, et al. Transitioning Between Prostanoid Therapies in Pulmonary Arterial Hypertension. Front Med. 2020.

\section{Lecturas recomendadas}

a. Hoeper MM, Benza RL, Corris P, de Perrot M, Fadel E, Keogh AM, et al. Intensive care, right ventricular support and lung transplantation in patients with pulmonary hypertension. Eur Respir J. 2019;53(1).

b. https://www.who.int/publications-detail/clinical-management-of-severe-acute-respiratory-infection-when-novel-coronavirus-(ncov)-infectionis-suspected) c. Padang R, Chandrashekar N, Indrabhinduwat M, Scott CG, Luis SA Chandrasekaran K, et al. Aetiology and outcomes of severe right ventricular dysfunction. Eur Heart J. 2020;41(12):1273-82.

d. Campo A, Mathai SC, Le Pavec J, Zaiman AL, Hummers LK, Boyce D, et al. Outcomes of hospitalisation for right heart failure in pulmonary arterial hypertension. Eur Respir J. 2011;38(2):359-67.

e. Wang D, Li S, Jiang J, Yan J, Zhao C, Wang Y, et al. Section of Precision Medicine Group of Chinese Society of Cardiology, Editorial Board of Chinese Journal of Cardiology, Working Group of Adult Fulminant Myocarditis (2019) Chinese society of cardiology expert consensus statement on the diagnosis and treatment of adult fulminant myocarditis. Sci China Life Sci 62(2):187-202.https://doi.org/10.1007/s1142 7-018-9385-3.

f. Piotr Ponikowski, Adriaan A Voors, Stefan D Anker, Héctor Bueno, John G F Cleland, Andrew J S Coats. ESC Scientific Document Group, 2016 ESC Guidelines for the diagnosis and treatment of acute and chronic heart failure: The Task Force for the diagnosis and treatment of acute and chronic heart failure of the European Society of Cardiology (ESC). Developed with the special contribution of the Heart Failure Association (HFA) of the ESC, European Heart Journal, Volume 37, Issue 27, 14 July 2016, Pages 2129-2200, https://doi.org/10.1093/eurheartj/ehw128. 